PROCEEDINGS OF THE

AMERICAN MATHEMATICAL SOCIETY

Volume 139, Number 2, February 2011, Pages 491-498

S 0002-9939(2010)10482-3

Article electronically published on July 12, 2010

\title{
ON THE DUALS OF SZEGÖ AND CAUCHY TUPLES
}

\author{
AMEER ATHAVALE AND PRAMOD PATIL
}

(Communicated by Nigel J. Kalton)

\begin{abstract}
The tuple of multiplications by coordinate functions on the Hardy space of the open unit ball $\mathbb{B}^{2 m}$ in $\mathbb{C}^{m}$ (resp. open unit polydisk $\mathbb{D}^{m}$ in $\mathbb{C}^{m}$ ) is referred to as the Szegö tuple (resp. Cauchy tuple) and is a well-known example of a subnormal operator tuple. Naturally associated with the Szegö tuple (resp. Cauchy tuple) is its dual whose coordinates act on the orthocomplement of the Hardy space of the ball (resp. polydisk) in an appropriate $L^{2}$ space. We examine the Koszul complexes associated with the duals of the Szegö and Cauchy tuples and determine their Betti numbers. We explicitly verify that, for $m \geq 2$, the $m^{\prime}$ th cohomology vector space associated with the Koszul complex of either the dual of the Szegö tuple or the dual of the Cauchy tuple is zero-dimensional. It follows in particular that, for $m \geq 2$, neither the Szegö $m$-tuple nor the Cauchy $m$-tuple is quasisimilar to its dual; this is in contrast with the case $m=1$ where both the Szegö tuple and the Cauchy tuple reduce to the Unilateral Shift, which is known to be unitarily equivalent to its dual.
\end{abstract}

\section{INTRODUCTION}

If $\mathcal{H}$ is a complex infinite-dimensional separable Hilbert space, then we use $\mathcal{B}(\mathcal{H})$ to denote the algebra of bounded linear operators on $\mathcal{H}$. An $m$-tuple $S=$ $\left(S_{1}, \cdots, S_{m}\right)$ of commuting operators $S_{i}$ in $\mathcal{B}(\mathcal{H})$ is said to be subnormal if there exist a Hilbert space $\mathcal{K}$ containing $\mathcal{H}$ and an $m$-tuple $N=\left(N_{1}, \cdots, N_{m}\right)$ of commuting normal operators $N_{i}$ in $\mathcal{B}(\mathcal{K})$ such that $N_{i} \mathcal{H} \subset \mathcal{H}$ and $N_{i} / \mathcal{H}=S_{i}$ for $1 \leq i \leq m$. Every subnormal operator tuple has a 'minimal' normal extension that is unique up to unitary equivalence (see [13). If $N=\left(N_{1}, \cdots, N_{m}\right)$ (with $N_{i}$ in $\mathcal{B}(\mathcal{K})$ ) is the minimal normal extension of a subnormal tuple $S=\left(S_{1}, \cdots, S_{m}\right)$ (with $S_{i}$ in $\mathcal{B}(\mathcal{H})$ ), and $\mathcal{H}^{\perp} \equiv \mathcal{K} \ominus \mathcal{H}$ is the orthocomplement of $\mathcal{H}$ in $\mathcal{K}$, then one defines the dual $\tilde{S}$ of $S$ to be the subnormal tuple $\tilde{S}=\left(\tilde{S}_{1}, \cdots, \tilde{S}_{m}\right)$, where $\tilde{S}_{i}=N_{i}^{*} / \mathcal{H}^{\perp}$. We note that $\left(\tilde{S}_{i}\right)^{*}=P_{\mathcal{H}^{\perp}} N_{i} / \mathcal{H}^{\perp}$, where $P_{\mathcal{H}^{\perp}}$ denotes the orthogonal projection of $\mathcal{K}$ onto $\mathcal{H}^{\perp}$.

If $\mathcal{H}^{2}\left(\mathbb{B}^{2 m}\right)$ denotes the Hardy space of the open unit ball $\mathbb{B}^{2 m}$ in $\mathbb{C}^{m}$, then we use $M_{\sigma, z}$ to denote the operator tuple $\left(M_{\sigma, z_{1}}, \cdots, M_{\sigma, z_{m}}\right)$ of multiplications by coordinate functions $z_{i}$ on $\mathcal{H}^{2}\left(\mathbb{B}^{2 m}\right)$ and refer to $M_{\sigma, z}$ as the Szegö tuple. If $\mathcal{H}^{2}\left(\mathbb{D}^{m}\right)$ denotes the Hardy space of the open unit polydisk $\mathbb{D}^{m}$ in $\mathbb{C}^{m}$, then we use $M_{\tau, z}$ to denote the operator tuple $\left(M_{\tau, z_{1}}, \cdots, M_{\tau, z_{m}}\right)$ of multiplications by coordinate functions $z_{i}$ on $\mathcal{H}^{2}\left(\mathbb{D}^{m}\right)$ and refer to $M_{\tau, z}$ as the Cauchy tuple. Here we have used

Received by the editors July 15, 2009 and, in revised form, March 3, 2010.

2010 Mathematics Subject Classification. Primary 47B20; Secondary 33C55.

Key words and phrases. Subnormal, quasisimilar, Koszul complex, spherical harmonics.

(C)2010 American Mathematical Society 
$\sigma$ to indicate the normalized 'surface area measure' on the unit sphere $\mathbb{S}^{2 m-1}$ and $\tau$ to indicate the normalized 'product arc-length measure' on $\mathbb{T}^{m}$, where $\mathbb{T}$ is the unit circle in $\mathbb{C}$. The Szegö tuple $M_{\sigma, z}$ and the Cauchy tuple $M_{\tau, z}$ are subnormal operator tuples. Indeed, the minimal normal extension of $M_{\sigma, z}$ is the multiplication tuple $N_{\sigma, z}$ on $L^{2}(\sigma)$ and that of $M_{\tau, z}$ is the multiplication tuple $N_{\tau, z}$ on $L^{2}(\tau)$. It may be noted that, for $m=1$, both the Szegö tuple and the Cauchy tuple reduce to the well-known Unilateral Shift.

Suppose $S=\left(S_{1}, \cdots, S_{m}\right)$ is a tuple of commuting operators in $\mathcal{B}(\mathcal{H})$ and $T=$ $\left(T_{1}, \cdots, T_{m}\right)$ a tuple of commuting operators in $\mathcal{B}(\mathcal{J})$. If there exists a bounded linear operator $X: \mathcal{H} \rightarrow \mathcal{J}$ such that $X S_{i}=T_{i} X$ for each $i$, then $X$ is said to be an intertwining operator (for $S$ and $T$ ) and we denote this fact by $X S=T X$. If $X: \mathcal{H} \rightarrow \mathcal{J}$ and $Y: \mathcal{J} \rightarrow \mathcal{H}$ are two intertwining operators for $S$ and $T$ such that $X S=T X$ and $Y T=S Y$, and both $X$ and $Y$ are injective and have dense ranges, then $S$ is said to be quasisimilar to $T$. The operator tuple $S$ is said to be unitarily equivalent to $T$ if one can find a unitary intertwining operator for $S$ and $T$.

The notion of the dual of a subnormal operator was introduced by Conway in [5], where he verified that the Unilateral Shift is self-dual, that is, unitarily equivalent to its dual. It would thus be natural to investigate the existence of special intertwining operators for the Szegö tuple and its dual and the Cauchy tuple and its dual. A particular consequence of the analysis to follow is that, for $m \geq 2$, neither the Szegö tuple nor the Cauchy tuple is even quasisimilar to its dual (see Proposition 2.2). The three basic ingredients needed for the proof of Proposition 2.2 are (i) the known spectral properties of the Szegö and Cauchy tuples, (ii) an examination of the long exact sequence $(* * *)$ of Section 2 associated with the short exact sequence $(* *)$ there, and (iii) the fact that quasisimilarity of certain subnormal tuples forces the equality of the corresponding Betti numbers of their Koszul complexes.

Let $e_{0}=1 \in \mathbb{C}$ and let $\left\{e_{1}, \cdots, e_{m}\right\}$ be the standard basis of $\mathbb{C}^{m}$. By the exterior algebra $\Gamma$ over $\mathbb{C}^{m}$ we understand the vector space direct sum $\Gamma=\Gamma^{0} \oplus \cdots \oplus \Gamma^{m}$, where $\Gamma^{0}=\mathbb{C}=\operatorname{lin}\left\{e_{0}\right\}$, where $\Gamma^{p}=\operatorname{lin}\left\{e_{i_{1}} \wedge \cdots \wedge e_{i_{p}}: 1 \leq i_{1}<\cdots<i_{p} \leq m\right\}$ $(1 \leq p \leq m)$, and where the multiplication $\wedge$ in $\Gamma$ is bilinear, associative, and satisfies the relations $1 \wedge \gamma=\gamma \wedge 1=\gamma(\gamma \in \Gamma), e_{i} \wedge e_{j}+e_{j} \wedge e_{i}=0(1 \leq i, j \leq m)$. One can think of $\Gamma$ as an orthogonal direct sum of Hilbert spaces with the inner product $\langle\cdot, \cdot\rangle_{p}$ on $\Gamma^{p}(p \geq 1)$ defined by $\left\langle e_{i_{1}} \wedge \cdots \wedge e_{i_{p}}, e_{j_{1}} \wedge \cdots \wedge e_{j_{p}}\right\rangle_{p}=\operatorname{det}\left(\left\langle e_{i_{k}}, e_{j_{l}}\right\rangle\right)$.

For $0 \leq i \leq m$, the operators $E_{i}$ on $\Gamma$ are defined by $E_{i} \gamma=e_{i} \wedge \gamma(\gamma \in \Gamma)$. Then $E_{0}$ is the identity operator on $\Gamma$, and the relations $E_{i} E_{j}+E_{j} E_{i}=0, E_{i} E_{j}^{*}+E_{j}^{*} E_{i}=$ $\delta_{i, j} E_{0}$ hold for $1 \leq i, j \leq m$.

Let $T$ be an $m$-tuple of commuting operators in $\mathcal{B}(\mathcal{J})$. Let $\Gamma(\mathcal{J})$ be the Hilbert space tensor product $\mathcal{J} \otimes \Gamma=\mathcal{J} \otimes \Gamma^{0} \oplus \cdots \oplus \mathcal{J} \otimes \Gamma^{m}\left(\equiv \Gamma^{0}(\mathcal{J}) \oplus \cdots \oplus \Gamma^{m}(\mathcal{J})\right)$, and let $\partial_{T}: \Gamma(\mathcal{J}) \rightarrow \Gamma(\mathcal{J})$ be defined by $\partial_{T}=\sum_{i=1}^{m} T_{i} \otimes E_{i}$. It is easy to check that $\partial_{T}^{2}=0$. The Koszul complex $K(T)$ is the cochain complex

$$
K(T): 0 \stackrel{\partial_{T,-1}}{\longrightarrow} \Gamma^{0}(\mathcal{J}) \stackrel{\partial_{T, 0}}{\longrightarrow} \Gamma^{1}(\mathcal{J}) \stackrel{\partial_{T, 1}}{\longrightarrow} \cdots \stackrel{\partial_{T, m-1}}{\longrightarrow} \Gamma^{m}(\mathcal{J}) \stackrel{\partial_{T, m}}{\longrightarrow} 0
$$

where $\partial_{T,-1}$ and $\partial_{T, m}$ are zero maps and $\partial_{T, p}(0 \leq p \leq m-1)$ are defined by $\partial_{T, p}=\partial_{T} / \Gamma^{p}(\mathcal{J})$.

For any tuple $T$ of commuting operators $T_{i}$ in $\mathcal{B}(\mathcal{J})$ and for any $\lambda=\left(\lambda_{1}, \cdots, \lambda_{m}\right)$ in $\mathbb{C}^{m}$, we use $T-\lambda$ to denote the tuple $\left(T_{1}-\lambda_{1} I_{\mathcal{J}}, \cdots, T-\lambda_{m} I_{\mathcal{J}}\right)$, where $I_{\mathcal{J}}$ stands for the identity operator on the Hilbert space $\mathcal{J}$. The Taylor spectrum $\sigma(T)$ is defined by $\sigma(T)=\left\{\lambda \in \mathbb{C}^{m}: K(T-\lambda)\right.$ is not exact $\}$. The coboundary maps 
$\partial_{T, p}$ give rise to the cohomology vector spaces $H^{(p)}(T)=\frac{\operatorname{Ker}\left(\partial_{T, p}\right)}{\operatorname{Ran}\left(\partial_{T, p-1}\right)}(0 \leq p \leq m)$. The tuple $T$ is said to be Fredholm if the Betti numbers $\beta_{p}(T)=\operatorname{dim}\left(H^{(p)}(T)\right)$ are all finite, and in that case the Fredholm index $\operatorname{ind}(T)$ of $T$ is defined to be the Euler characteristic of $K(T)$, viz. $\operatorname{ind}(T)=\sum_{p=0}^{m}(-1)^{p} \beta_{p}$. The essential Taylor spectrum $\sigma_{\text {ess }}(T)$ of $T$ is the set $\sigma_{\text {ess }}(T)=\left\{\lambda \in \mathbb{C}^{m}: T-\lambda\right.$ is not Fredholm $\}$. Both $\sigma(T)$ and $\sigma_{\text {ess }}(T)$ are non-empty compact subsets of $\mathbb{C}^{m}$ with $\sigma_{\text {ess }}(T) \subset \sigma(T)$. The tuple $T$ is said to be invertible if the complex $(*)$ is exact, that is, if $\beta_{p}(T)=0$ for all $p$. Clearly, every invertible tuple $T$ is Fredholm. For $T$ Fredholm, the coboundary maps $\partial_{T, p}$ have closed ranges, and $T=\left(T_{1}, \ldots, T_{m}\right)$ is Fredholm if and only if $T^{*}=\left(T_{1}^{*}, \ldots, T_{m}^{*}\right)$ is. The basic properties of $\sigma(T)$ and $\sigma_{e s s}(T)$ are discussed in [6] and [8] (using chain complexes rather than cochain ones).

At this stage, we find it necessary to record a few observations related to the chain version of the Koszul complex as considered in 6 . We first note that the $p^{\prime}$ th homology vector space of the chain Koszul complex in [6] is isomorphic to the $(m-p)$ 'th cohomology vector space $H^{(m-p)}(T)$ as defined here; in particular, using $\gamma_{p}(T)$ to denote the $p$ 'th Betti number of the chain Koszul complex of [6], we have $\gamma_{p}(T)=\beta_{m-p}(T)$. In [6], Curto provides an algorithm whereby one can associate with $T$ an operator matrix $\hat{T}$ of order $2^{m-1}$ acting on the $2^{m-1}$-fold ampliation of $\mathcal{J}$ in such a way that $T$ is invertible (resp. Fredholm) as an operator tuple if and only if $\hat{T}$ is invertible (resp. Fredholm) as a single operator. For example, for $m=2$,

and, for $m=3$,

$$
\hat{T}=\left[\begin{array}{cc}
T_{1} & T_{2} \\
-T_{2}^{*} & T_{1}^{*}
\end{array}\right]
$$

$$
\hat{T}=\left[\begin{array}{cccc}
T_{1} & T_{2} & T_{3} & 0 \\
-T_{2}^{*} & T_{1}^{*} & 0 & T_{3} \\
-T_{3}^{*} & 0 & T_{1}^{*} & -T_{2} \\
0 & -T_{3}^{*} & T_{2}^{*} & T_{1}
\end{array}\right] .
$$

As observed in [6] (refer in particular to Theorem 4 there), if $T$ is Fredholm, then $\hat{T}(\hat{T})^{*}$ is a block-diagonal matrix $\operatorname{diag}\left[E_{0}(T), E_{2}(T), \ldots\right]$ of $\left[\frac{m}{2}\right]+1$ matrix entries $E_{2 k}(T)$, where each $E_{2 k}(T)$ is of order $\left(\begin{array}{c}m \\ 2 k\end{array}\right)$ with $\operatorname{dim}\left(\operatorname{Ker}\left(E_{2 k}(T)\right)\right)=\gamma_{2 k}(T)$, and $(\hat{T})^{*} \hat{T}$ is a block-diagonal matrix $\operatorname{diag}\left[F_{1}(T), F_{3}(T), \ldots\right]$ of $\left[\frac{m-1}{2}\right]+1$ matrix entries $F_{2 k+1}(T)$, where each $F_{2 k+1}(T)$ is of order $\left(\begin{array}{c}m \\ 2 k+1\end{array}\right)$ with $\operatorname{dim}\left(\operatorname{Ker}\left(F_{2 k+1}(T)\right)\right)=$ $\gamma_{2 k+1}(T)$. Using $\simeq$ to indicate unitary equivalence, we observe that $E_{2 k}(T) \simeq$ $E_{m-2 k}\left(T^{*}\right), \quad F_{2 k+1}(T) \simeq F_{m-(2 k+1)}\left(T^{*}\right)$ in case $m$ is even, and $E_{2 k}(T)$ $\simeq F_{m-2 k}\left(T^{*}\right), F_{2 k+1}(T) \simeq E_{m-(2 k+1)}\left(T^{*}\right)$ in case $m$ is odd. Indeed, the unitary equivalence $\simeq$ is actually an equality in case the two matrices are of order 1 ; otherwise, $\simeq$ is induced by a cross-diagonal matrix with some of the entries on the cross-diagonal being the identity operator $I_{\mathcal{J}}$ and some of them being $-I_{\mathcal{J}}$. As a consequence, one has $\beta_{p}(T)=\gamma_{m-p}(T)=\gamma_{p}\left(T^{*}\right)=\beta_{m-p}\left(T^{*}\right)$ for a Fredholm $m$-tuple $T$.

\section{Duals of Szegö and Cauchy tuples}

Using $\bar{A}$ to denote the closure of a set $A$ in $\mathbb{C}^{m}$, it follows from the results in [6] that $\sigma\left(N_{\sigma, z}\right)=\sigma_{e s s}\left(N_{\sigma, z}\right)=\mathbb{S}^{2 m-1}, \sigma\left(M_{\sigma, z}\right)=\overline{\mathbb{B}^{2 m}}, \sigma_{e s s}\left(M_{\sigma, z}\right)=\mathbb{S}^{2 m-1}$, $\sigma\left(N_{\tau, z}\right)=\sigma_{e s s}\left(N_{\tau, z}\right)=\mathbb{T}^{m}, \sigma\left(M_{\tau, z}\right)=\overline{\mathbb{D}^{m}}, \sigma_{e s s}\left(M_{\tau, z}\right)=\partial\left(\mathbb{D}^{m}\right)\left(\right.$ with $\partial\left(\mathbb{D}^{m}\right)$ standing for the topological boundary of $\left.\mathbb{D}^{m}\right)$; furthermore, for any $\lambda \in \mathbb{B}^{2 m}$, 
$\operatorname{ind}\left(M_{\sigma, z}-\lambda\right)=(-1)^{m}$ and, for any $\lambda \in \mathbb{D}^{m}$, ind $\left(M_{\tau, z}-\lambda\right)=(-1)^{m}$. In [1, it was conjectured that, in case both a subnormal tuple $S$ and its dual $\tilde{S}$ happen to be Fredholm, one has ind $(\tilde{S})=(-1)^{(m+1)} \operatorname{ind}(S)$. A few years ago, R. E. Curto confirmed in a private communication to the first-named author that the conjecture was correct (refer to [2]); recently it was verified in a swift manner by Gleason in [11] by considering the exact sequence of Koszul complexes

$$
0 \longrightarrow K(S) \longrightarrow K(N) \longrightarrow K\left((\tilde{S})^{*}\right) \longrightarrow 0,
$$

where the arrow between $K(S)$ and $K(N)$ is induced by the inclusion of $\mathcal{H}$ into $\mathcal{K}$ and where the arrow between $K(N)$ and $K\left((\tilde{S})^{*}\right)$ is induced by the orthogonal projection $P_{\mathcal{H}^{\perp}}$ of $\mathcal{K}$ onto $\mathcal{H}^{\perp}$. It follows from some general theory developed in [4] and the sequence $(* *)$ that, given $S$ to be Fredholm, $(\tilde{S})^{*}$ (equivalently $\tilde{S}$ ) is Fredholm if and only if $N$ is. It is then clear that both $\tilde{M}_{\sigma, z}$ and $\tilde{M}_{\tau, z}$ are Fredholm; in particular, the coboundary maps associated with $\tilde{M}_{\sigma, z}$ and $\tilde{M}_{\tau, z}$ have closed ranges.

The proof of Proposition 2.1 below can be provided appealing to the exact sequence $(* *)$ and using the same arguments as in [6] (refer also to [1, 9] and [10); we omit the proof.

Proposition 2.1. (a) $\sigma\left(\tilde{M}_{\sigma, z}\right)=\overline{\mathbb{B}^{2 m}}, \sigma_{e s s}\left(\tilde{M}_{\sigma, z}\right)=\mathbb{S}^{2 m-1}$, and $\operatorname{ind}\left(\tilde{M}_{\sigma, z}-\lambda\right)=$ -1 for every $\lambda \in \mathbb{B}^{2 m}$.

(b) $\sigma\left(\tilde{M}_{\tau, z}\right)=\overline{\mathbb{D}^{m}}, \sigma_{e s s}\left(\tilde{M}_{\tau, z}\right)=\partial\left(\mathbb{D}^{m}\right)$, and $\operatorname{ind}\left(\tilde{M}_{\tau, z}-\lambda\right)=-1$ for every $\lambda \in \mathbb{D}^{m}$.

The short exact sequence $(* *)$ gives rise to the long exact sequence of cohomology

$$
\begin{aligned}
& (* * *) \\
& \quad 0 \rightarrow H^{(0)}(S) \rightarrow H^{(0)}(N) \rightarrow H^{(0)}\left((\tilde{S})^{*}\right) \rightarrow H^{(1)}(S) \rightarrow H^{(1)}(N) \rightarrow H^{(1)}\left((\tilde{S})^{*}\right) \rightarrow H^{(2)}(S) \rightarrow \ldots \\
& \quad \rightarrow H^{(m-1)}(S) \rightarrow H^{(m-1)}(N) \rightarrow H^{(m-1)}\left((\tilde{S})^{*}\right) \rightarrow H^{(m)}(S) \rightarrow H^{(m)}(N) \rightarrow H^{(m)}\left((\tilde{S})^{*}\right) \rightarrow 0 .
\end{aligned}
$$

It is known that $\beta_{p}\left(M_{\sigma, z}\right)=\beta_{p}\left(M_{\tau, z}\right)=1$ if $p=m$, and $\beta_{p}\left(M_{\sigma, z}\right)=\beta_{p}\left(M_{\tau, z}\right)=0$ if $p \neq m$ (refer, for example, to [12]). Also, $H^{(p)}\left(N_{\sigma, z}\right)$ and $H^{(p)}\left(N_{\tau, z}\right)$ are zerodimensional for all $p$. It follows then from $(* * *)$ that, for $S$ either the Szegö tuple $M_{\sigma, z}$ or the Cauchy tuple $M_{\tau, z}, \beta_{p}\left((\tilde{S})^{*}\right)=1$ if $p=m-1$, and $\beta_{p}\left((\tilde{S})^{*}\right)=0$ if $p \neq m-1$; in the light of our comments at the end of Section 1, this further leads to $\beta_{p}(\tilde{S})=1$ if $p=1$, and $\beta_{p}(\tilde{S})=0$ if $p \neq 1$. In particular, our observation in Proposition 2.1 that $\operatorname{ind}\left(M_{\sigma, z}\right)=\operatorname{ind}\left(M_{\tau, z}\right)=-1$ stands sharpened.

Proposition 2.2. For $m \geq 2$, neither the Szegö $m$-tuple $M_{\sigma, z}$ nor the Cauchy m-tuple $M_{\tau, z}$ is quasisimilar to its dual.

Proof. An examination of the proof of Theorem 2 in [3] (which relies upon Lemma 1 in [3) reveals the following: If a subnormal operator tuple $T$ is quasisimilar to the Szegö tuple $S=M_{\sigma, z}$, then $T$ must be Fredholm and the dimension of any cohomology vector space $H^{(p)}(T)$ must be the same as that of $H^{(p)}(S)$ (that is, $\beta_{p}(T)=\beta_{p}(S)$ for all $\left.p\right)$. For $m \geq 2, \beta_{p}(S)$ differs from $\beta_{p}(\tilde{S})$ for $p=1$ and $p=m$, and the desired result is obvious. As was pointed out in [3], the proof of Theorem 2 in $\left[3\right.$. (which was presented in the context of the ball $\mathbb{B}^{2 m}$ ) can also be adapted to the context of the polydisk $\mathbb{D}^{m}$. In particular, one has the following: If a subnormal operator tuple $T$ is quasisimilar to the Cauchy tuple $M_{\tau, z}$, then $T$ must be Fredholm and $\beta_{p}(T)=\beta_{p}\left(M_{\tau, z}\right)$ for all $p$. One now argues exactly as in the case of the Szegö tuple. 
Let us suppose that the operator tuple $T$ of the complex $(*)$ is either $\tilde{M}_{\sigma, z}$ or $\tilde{M}_{\tau, z}$ (with the underlying Hilbert space $\mathcal{J}$ being either the orthocomplement $\mathcal{H}^{\perp}$ of $\mathcal{H}=\mathcal{H}^{2}\left(\mathbb{B}^{2 m}\right)$ in $L^{2}(\sigma)$ or the orthocomplement $\mathcal{H}^{\perp}$ of $\mathcal{H}=\mathcal{H}^{2}\left(\mathbb{D}^{m}\right)$ in $\left.L^{2}(\tau)\right)$. The coboundary map $\partial_{T, 0}$ is given by $f \mapsto\left(T_{1} f, T_{2} f, \ldots, T_{m} f\right.$ ), $f \in \mathcal{J}$ (where the action of $T_{i}$ is multiplication by $\left.\bar{z}_{i}\right)$. It is trivial to see that $\partial_{T, 0}$ has zero-dimensional kernel. The coboundary map $\partial_{T, 1}$ is given by $\left(f_{1}, f_{2}, \ldots, f_{m}\right) \mapsto\left(T_{1} f_{2}-T_{2} f_{1}, T_{1} f_{3}-\right.$ $\left.T_{3} f_{1}, \ldots, T_{1} f_{m}-T_{m} f_{1}, T_{2} f_{3}-T_{3} f_{2}, \ldots, T_{m-1} f_{m}-T_{m} f_{m-1}\right), f_{i} \in \mathcal{J}$. We note that the vector space $H^{(1)}(T)$ is isomorphic to $\operatorname{Ker}\left(\partial_{T, 1}\right) \cap\left(\operatorname{Ran}\left(\partial_{T, 0}\right)\right)^{\perp}$. Clearly, the vector $\left(\bar{z}_{1}, \ldots, \bar{z}_{m}\right)$ lies in $\operatorname{Ker}\left(\partial_{T, 1}\right)$, and, for any $f$ in $\mathcal{J}$, its inner product with $\left(\bar{z}_{1} f, \ldots, \bar{z}_{m} f\right)$ equals $\langle f, 1\rangle_{L^{2}(\sigma)}=0$ in case $T=\tilde{M}_{\sigma, z}$ and $m\langle f, 1\rangle_{L^{2}(\tau)}=0$ in case $T=\tilde{M}_{\tau, z}$. Thus the 1 -dimensional vector space $\operatorname{Ker}\left(\partial_{T, 1}\right) \cap\left(\operatorname{Ran}\left(\partial_{T, 0}\right)\right)^{\perp}$ is spanned by $\left(\bar{z}_{1}, \ldots, \bar{z}_{m}\right)$.

In Sections 3 and 4 below we provide explicit analytic proofs of the fact that, for $m \geq 2$ and for $S$ either the Szegö tuple or the Cauchy tuple, the $m$ 'th cohomolgy vector space $H^{(m)}(\tilde{S})=\frac{\mathcal{H}^{\perp}}{\operatorname{Ran} \partial_{\tilde{S}, m-1}}=\frac{\mathcal{H}^{\perp}}{\left(\tilde{S}_{1} \mathcal{H}^{\perp}+\ldots+\tilde{S}_{m} \mathcal{H}^{\perp}\right)}$ associated with the Koszul complex $K(\tilde{S})$ of $\tilde{S}$ is zero-dimensional, that is, $\beta_{m}(\tilde{S})=0$. In the case of the dual of the Szegö tuple, that effort rests on the use of complex spherical harmonics.

\section{Dual of Szegö tuple}

In the present section we assume $m \geq 2$. A polynomial $f(z, \bar{z})$ in the variables $z$ and $\bar{z}$ (with $z=\left(z_{1}, \ldots, z_{m}\right) \in \mathbb{C}^{m}$ ) is said to be harmonic if $\sum_{j=1}^{m} \frac{\partial^{2} f}{\partial z_{j} \partial \bar{z}_{j}}(z, \bar{z})=0$ for all $z$ in $\mathbb{C}^{m}$. A complex spherical harmonic is the restriction of a harmonic polynomial $f(z, \bar{z})$ to $\mathbb{S}^{2 m-1}$. For any non-negative integers $p$ and $q$, we use $H(p, q)$ to denote the set of all complex spherical harmonics that are the restrictions to $\mathbb{S}^{2 m-1}$ of those harmonic polynomials $f(z, \bar{z})$ that are homogeneous of bidegree $(p, q)$ in $z$ and $\bar{z}$. The spaces $H(p, q)$ are finite-dimensional pairwise orthogonal subspaces of $L^{2}(\sigma)$ (refer to Section 12 of [14). As Theorem 12.2.3 of [14] states,

$$
L^{2}(\sigma)=\bigoplus_{p \geq 0, q \geq 0} H(p, q)
$$

where $\oplus$ denotes the (closed) direct sum. It is clear from (A) that $H^{2}\left(\mathbb{B}^{2 m}\right)=$ $\bigoplus_{p \geq 0} H(p, 0)$ and $H^{2}\left(\mathbb{B}^{2 m}\right)^{\perp}=\bigoplus_{p \geq 0, q \geq 1} H(p, q)$. A special consequence of Theorem 12.4.4 of [14] is that, given $p \geq 0$ and $q \geq 1, z_{i} H(p, q) \subset H(p+1, q)+H(p, q-1)$ for $1 \leq i \leq m$. form

Any element of $H(p, 1)$ is the restriction to $\mathbb{S}^{2 m-1}$ of a polynomial $f(z, \bar{z})$ of the

$$
f(z, \bar{z})=\sum_{i=1}^{m} \bar{z}_{i} f_{i}(z),
$$

where $f_{i}$, if non-zero, is a homogeneous polynomial of degree $p$. The polynomials $f_{i}$, being either zero or homogeneous of degree $p$, satisfy

$$
\sum_{j=1}^{m} z_{j} \frac{\partial f_{i}}{\partial z_{j}}(z)=p f_{i}(z) ;
$$


further, $f(z, \bar{z})$ is harmonic so that the $f_{i}$ also satisfy

$$
\sum_{i=1}^{m} \frac{\partial f_{i}}{\partial z_{i}}(z)=0
$$

The most crucial part of our analysis in the present section is checking that any element of $H(p, 1)$ can be written as $\sum_{i=1}^{m} \bar{z}_{i} g_{i}(z, \bar{z})$, where each $g_{i}$ is in $H(p+1,1)$. To illustrate, we consider the case $m=2$. Modulo $\left|z_{1}\right|^{2}+\left|z_{2}\right|^{2}=1, \bar{z}_{1}$ (whose restriction to $\mathbb{S}^{2 m-1}$ is in $\left.H(0,1)\right)$ can be written as $\bar{z}_{1}=\bar{z}_{1}\left(z_{1} \bar{z}_{1}-z_{2} \bar{z}_{2}\right)+\bar{z}_{2}\left(2 z_{2} \bar{z}_{1}\right)$; it should be noted that $z_{1} \bar{z}_{1}-z_{2} \bar{z}_{2}$ and $2 z_{2} \bar{z}_{1}$ are harmonic polynomials that are homogeneous of bidegree $(1,1)$. Also, $z_{2} \bar{z}_{1}$ and $z_{1} \bar{z}_{1}-z_{2} \bar{z}_{2}$ (whose restrictions to $\mathbb{S}^{2 m-1}$ are in $\left.H(1,1)\right)$ can, modulo $\left|z_{1}\right|^{2}+\left|z_{2}\right|^{2}=1$, be written as $z_{2} \bar{z}_{1}=\bar{z}_{1}\left(z_{1} z_{2} \bar{z}_{1}-\right.$ $\left.\frac{1}{2} z_{2}^{2} \bar{z}_{2}\right)+\bar{z}_{2}\left(\frac{3}{2} z_{2}^{2} \bar{z}_{1}\right)$ and $z_{1} \bar{z}_{1}-z_{2} \bar{z}_{2}=\bar{z}_{1}\left(z_{1}^{2} \bar{z}_{1}-2 z_{1} z_{2} \bar{z}_{2}\right)+\bar{z}_{2}\left(2 z_{1} z_{2} \bar{z}_{1}-z_{2}^{2} \bar{z}_{2}\right)$; it should be noted that $z_{1} z_{2} \bar{z}_{1}-\frac{1}{2} z_{2}^{2} \bar{z}_{2}, \frac{3}{2} z_{2}^{2} \bar{z}_{1}, z_{1}^{2} \bar{z}_{1}-2 z_{1} z_{2} \bar{z}_{2}, 2 z_{1} z_{2} \bar{z}_{1}-z_{2}^{2} \bar{z}_{2}$ are harmonic polynomials that are homogeneous of bidegree $(2,1)$. The general result is given by the following lemma.

Lemma 3.1. For $p \geq 0$, any element of $H(p, 1)$ can be written as $\sum_{i=1}^{m} \bar{z}_{i} g_{i}(z, \bar{z})$, where each $g_{i}$ is in $H(p+1,1)$.

Proof. Let an element of $H(p, 1)$ be given by the restriction to $\mathbb{S}^{2 m-1}$ of $f(z, \bar{z})=$ $\sum_{i=1}^{m} \bar{z}_{i} f_{i}(z)$ as in (B). On $\mathbb{S}^{2 m-1}, f(z, \bar{z})$ can be rewritten as

$$
\begin{aligned}
f(z, \bar{z})= & \bar{z}_{1}\left\{z_{1} f_{1}(z) \bar{z}_{1}-\frac{1}{m+p-1}\left(\sum_{i \neq 1} z_{i} \bar{z}_{i}\right) f_{1}(z)\right. \\
& \left.+\frac{m+p}{m+p-1} z_{1} \sum_{i \neq 1} f_{i}(z) \bar{z}_{i}\right\} \\
+ & \bar{z}_{2}\left\{z_{2} f_{2}(z) \bar{z}_{2}-\frac{1}{m+p-1}\left(\sum_{i \neq 2} z_{i} \bar{z}_{i}\right) f_{2}(z)\right. \\
+ & \left.\frac{m+p}{m+p-1} z_{2} \sum_{i \neq 2} f_{i}(z) \bar{z}_{i}\right\} \\
+\ldots+\bar{z}_{m}\left\{z_{m} f_{m}(z) \bar{z}_{m}\right. & -\frac{1}{m+p-1}\left(\sum_{i \neq m} z_{i} \bar{z}_{i}\right) f_{m}(z) \\
& \left.+\frac{m+p}{m+p-1} z_{m} \sum_{i \neq m} f_{i}(z) \bar{z}_{i}\right\} \\
\equiv & \bar{z}_{1} g_{1}(z, \bar{z})+\bar{z}_{2} g_{2}(z, \bar{z})+\ldots+\bar{z}_{m} g_{m}(z, \bar{z}) .
\end{aligned}
$$

Clearly each $g_{i}$, if non-zero, is a homogeneous polynomial of bidegree $(p+1,1)$. Using $(\mathrm{C})$ and $(\mathrm{D})$, one verifies in a straightforward way that each $g_{i}$ is harmonic.

Proposition 3.2. For $m \geq 2$, the $m$ 'th cohomology vector space $H^{(m)}(\tilde{S})$ associated with the Koszul complex $K(\tilde{S})$ of the dual $\tilde{S}$ of $S=M_{\sigma, z}$ is zero-dimensional, that is, $\beta_{m}(\tilde{S})=0$.

Proof. We first observe that the action of $\tilde{S}_{i}$ on $\mathcal{H}^{\perp}=H^{2}\left(\mathbb{B}^{2 m}\right)^{\perp}$ is multiplication by $\bar{z}_{i}$. It follows then from Lemma 3.1 that, for any $p \geq 0, H(p, 1) \subset \operatorname{Ran}\left(\partial_{\tilde{S}, m-1}\right)$ (where $\partial_{\tilde{S}, m-1}$ is the $(m-1)$ 'th coboundary operator of $K(\tilde{S})$ with closed range). 
Thus one has $\bigcap_{i=1}^{m} \operatorname{Ker}\left(\left(\tilde{S}_{i}\right)^{*}\right)=\mathcal{H}^{\perp} \ominus \operatorname{Ran}\left(\partial_{\tilde{S}, m-1}\right) \subset \mathcal{H}^{\perp} \ominus\left(\bigoplus_{p>0} H(p, 1)\right)=$ $\bigoplus_{p \geq 0, q \geq 2} H(p, q)$. Let $f \in \bigcap_{i=1}^{m} \operatorname{Ker}\left(\left(\tilde{S}_{i}\right)^{*}\right)$. Since $\left(\tilde{S}_{i}\right)^{*}=P_{\mathcal{H}^{\perp}} N_{\sigma, z_{i}} / \mathcal{H}^{\perp}$ and since the action of $N_{\sigma, z_{i}}$ on $L^{2}(\sigma)$ is multiplication by $z_{i}$, we have $P_{\mathcal{H}^{\perp}} z_{i} f=0$ for all $i$. But $f \in \bigoplus_{p \geq 0, q \geq 2} H(p, q)$ so that $z_{i} f \in \bigoplus_{p \geq 0, q \geq 1} H(p, q)=H^{2}\left(\mathbb{B}^{2 m}\right)^{\perp}$ in view of $z_{i} H(p, q) \subset H(p+1, q)+H(p, q-1)$. Thus $z_{i} f=P_{\mathcal{H}^{\perp}} z_{i} f=0$ for all $i$, and this clearly implies that $f$ is zero.

\section{Dual of Cauchy tuple}

As in Section 3, we assume $m \geq 2$. The functions $z_{1}^{n_{1}} z_{2}^{n_{2}} \ldots z_{m}^{n_{m}}$ ( $n_{i}$ integers), thought of as defined on the torus $\mathbb{T}^{m}$, form an orthogonal basis of $L^{2}(\tau)$. For $A \subset L^{2}(\tau)$, we use $\operatorname{cls} A$ to denote the closed linear span of $A$ in $L^{2}(\tau)$.

Proposition 4.1. For $m \geq 2$, the $m$ 'th cohomology vector space $H^{(m)}(\tilde{S})$ associated with the Koszul complex $K(\tilde{S})$ of the dual $\tilde{S}$ of $S=M_{\tau, z}$ is zero-dimensional, that is, $\beta_{m}(\tilde{S})=0$.

Proof. We present the proof for the case $m=2$; it is hardly different in the general case. If $\mathcal{H}=H^{2}\left(\mathbb{D}^{2}\right)$ and $\mathcal{K}=L^{2}(\tau)$, then $\mathcal{H}^{\perp}=H^{2}\left(\mathbb{D}^{2}\right)^{\perp}=\mathcal{H}_{1} \oplus \mathcal{H}_{2} \oplus \mathcal{H}_{3}$, where $\mathcal{H}_{1}=\operatorname{cls}\left\{\bar{z}_{1}^{p} z_{2}^{q}: p \geq 1, q \geq 0\right\}, \mathcal{H}_{2}=\operatorname{cls}\left\{z_{1}^{p} \bar{z}_{2}^{q}: p \geq 0, q \geq 1\right\}$, and $\mathcal{H}_{3}=\operatorname{cls}\left\{\bar{z}_{1}^{p} \bar{z}_{2}^{q}: p \geq 1, q \geq 1\right\}$. Let $f \in \bigcap_{i=1}^{2} \operatorname{Ker}\left(\left(\tilde{S}_{i}\right)^{*}\right)=\mathcal{H}^{\perp} \ominus \operatorname{Ran}\left(\partial_{\tilde{S}, 1}\right)$. (It may be recalled that $\partial_{\tilde{S}, 1}$ has closed range.) We write $f=\sum_{p \geq 1, q \geq 0} a_{p, q} \bar{z}_{1}^{p} z_{2}^{q}+$ $\sum_{p \geq 0, q \geq 1} b_{p, q} z_{1}^{p} \bar{z}_{2}^{q}+\sum_{p \geq 1, q \geq 1} c_{p, q} \bar{z}_{1}^{p} \bar{z}_{2}^{q}$ for some scalars $a_{p, q}, b_{p, q}, c_{p, q}$. Since $\left(\tilde{S}_{i}\right)^{*}=$ $P_{\mathcal{H}^{\perp}} N_{\tau, z_{i}} / \mathcal{H}^{\perp}$ and since the action of $N_{\tau, z_{i}}$ on $L^{2}(\tau)$ is multiplication by $z_{i}$, we have $P_{\mathcal{H} \perp} z_{i} f=0$ for $i=1,2$. In particular, utilizing $\left|z_{1}\right|=1$, one obtains $0=P_{\mathcal{H} \perp} z_{1} f=$ $\sum_{p \geq 2, q \geq 0} a_{p, q} \bar{z}_{1}^{p-1} z_{2}^{q}+\sum_{p \geq 0, q \geq 1} b_{p, q} z_{1}^{p+1} \bar{z}_{2}^{q}+\sum_{p \geq 1, q \geq 1} c_{p, q} \bar{z}_{1}^{p-1} \bar{z}_{2}^{q}$. This leads to $a_{p, q}=0(p \geq 2, q \geq 0), b_{p, q}=0(p \geq 0, q \geq 1)$, and $c_{p, q}=0(p \geq 1, q \geq 1)$, forcing $f=\sum_{q \geq 0} a_{1, q} \bar{z}_{1} z_{2}^{q}$. But then the equalities $0=P_{\mathcal{H}^{\perp}} z_{2} f=\sum_{q \geq 0} a_{1, q} \bar{z}_{1} z_{2}^{q+1}$ further force $a_{1, q}=0(q \geq 0)$ yielding $f=0$.

We conclude by emphasizing that neither the analysis of Section 3 nor that of Section 4 is possible unless $m \geq 2$.

\section{REFERENCES}

[1] A. Athavale, On the duals of subnormal tuples, Integral Equations and Operator Theory 12 (1989), 305-323. MR.998276 (90g:47042)

[2] A. Athavale, On the intertwining of joint isometries, J. Operator Theory 23 (1990), 339-350. MR.1066811 (91i:47029)

[3] A. Athavale, Quasisimilarity-invariance of joint spectra for certain subnormal tuples, Bull. London Math. Soc. 5 (2008), 759-769. MR2439641 (2009j:47038)

[4] C.-G. Ambrozie and F.-H. Vasilescu, Banach Space Complexes, Kluwer Academic Publications, Dordrecht, 1995. MR.1357165 (97a:47001)

[5] J. B. Conway, The dual of a subnormal operator, J. Operator Theory 5 (1981), 195-211. MR617974 (84j:47037)

[6] R. E. Curto, Fredholm and invertible n-tuples of operators. The deformation problem, Trans. Amer. Math. Soc. 266 (1981), 129-159. MR613789 (82g:47010)

[7] R. E. Curto, Spectral inclusion for doubly commuting subnormal n-tuples, Proc. Amer. Math. Soc. 83 (1981), 730-734. MR630045 (82j:47030)

[8] R. E. Curto, Applications of several complex variables to multiparameter spectral theory, Surveys of some recent results in operator theory, Vol. II, eds. J. B. Conway and B. B. Morrel, Pitman Research Notes in Mathematics Series, Longman Scientific and Technical, UK (1988), 25-90. MR976843 (90d:47007) 
[9] R. E. Curto and K. Yan, The spectral picture of Reinhardt measures, J. Func. Anal. 131 (1995), 279-301. MR1345033 (96i:47006)

[10] J. Eschmeier and M. Putinar, Spectral decompositions and analytic sheaves, London Mathematical Series Monographs, New Series, vol. 10, Clarendon Press, Oxford (1996). MR.1420618 (98h:47002)

[11] J. Gleason, On a question of Ameer Athavale, Irish Math. Soc. Bull. 48 (2002), 31-33. MR:1930523 (2003h:47041)

[12] J. Gleason, S. Richter and C. Sundberg, On the index of invariant subspaces in spaces of analytic functions in several complex variables, J. Reine Angew. Math. 587 (2005), 49-76. MR2186975 (2006i:47013)

[13] T. Ito, On the commutative family of subnormal operators, J. Fac. Sci. Hokkaido Univ. 14 (1958), 1-15. MR0107177 (21:5902)

[14] W. Rudin, Function theory in the unit ball of $\mathbb{C}^{n}$, Springer-Verlag, New York, 1980. MR601594 (82i:32002)

Department of Mathematics, Indian Institute of Technology Bombay, Powai, Mumbai 400076, INDIA

E-mail address: athavale@math.iitb.ac.in

Department of Mathematics, Indian Institute of Technology Bombay, Powai, Mumbai 400076, INDIA

E-mail address: pramodp@math.iitb.ac.in 